\title{
NCO-sP(EO-stat-PO) surface coatings preserve biochemical properties of RGD peptides
}

\author{
JÖRG FIEDLER ${ }^{1 *}$, JÜRGEN GROLL ${ }^{2 *}$, ERIKA ENGELHARDT $^{1}$, PETER GASTEIER $^{2}$, CLAUDIA DAHMEN $^{3}$, \\ HORST KESSLER $^{3}$, MARTIN MOELLER $^{2}$ and ROLF E. BRENNER ${ }^{1}$ \\ ${ }^{1}$ Orthopaedic Clinic, Division for Biochemistry of Joint and Connective Tissue Diseases, University of Ulm, \\ Oberer Eselsberg 45, D-89081 Ulm; ${ }^{2}$ DWI at the RWTH Aachen, Pauwelsstr. 8, D-52074 Aachen; \\ ${ }^{3}$ Institute for Advanced Study and Center of Integrated Protein Structure at the Technische \\ Universität München, Department Chemie, Lichtenbergstr. 4, D-85747 Garching, Germany
}

Received August 16, 2010; Accepted October 1, 2010

DOI: $10.3892 / \mathrm{ijmm} .2010 .553$

\begin{abstract}
We have previously reported that star shaped poly(ethylene oxide-stat-propylene oxide) macromers with $80 \%$ EO content and isocyanate functional groups at the distal ends [NCO-sP(EO-stat-PO)] can be used to generate coatings that are non-adhesive but easily functionalized for specific cell adhesion. In the present study, we investigated whether the NCO-sP(EO-stat-PO) surfaces maintain peptide configuration-specific cell-surface interactions or if differences between dissimilar binding molecules are concealed by the coating. To this end, we have covalently immobilized both linear-RGD peptides (gRGDsc) and cyclic-RGD (RGDfK) peptides in such coatings. Subsequently, SaOS-2 or human multipotent mesenchymal stromal cells (MSC) were seeded on these substrates. Cell adhesion, spreading and survival was observed for up to 30 days. The time span for cell adherence was not different on linear and cyclic RGD peptides, but was shorter in comparison to the unmodified glass surface. MSC proliferation on cyclic RGDfK modified coatings was 4 times higher than on films functionalized by linear gRGDsc sequences, underlining that the NCO-sP(EO-stat-PO) film preserves the configuration-specific biochemical peptide properties. Under basal conditions, MSC expressed osteogenic marker genes after 14 days on cyclic RGD peptides, but not on linear RGD peptides or the unmodified glass surfaces.
\end{abstract}

Correspondence to: Dr Rolf E. Brenner, Division for Biochemistry of Joint and Connective Tissue Diseases, Department of Orthopaedics, University of Ulm, Oberer Eselsberg 45, D-89081 Ulm, Germany

E-mail: rolf.brenner@uni-ulm.de

${ }^{*}$ Contributed equally

Abbreviations: NCO-sP(EO-stat-PO), NCO-functional star shaped poly(ethylene oxide-stat-propylene oxide) macromers with $80 \%$ EO content; PCR, polymerase chain reaction; RT-PCR, reverse transcription polymerase chain reaction

Key words: multipotent mesenchymal stromal cell, RGD peptide, surface coating
Our results indicate specific effects of these adhesion peptides on MSC biology and show that this coating system is useful for selective testing of cellular interactions with adhesive ligands.

\section{Introduction}

The surface design of biomaterials is a key element controlling their integration into the surrounding tissue and cell adhesion (1). The biomaterial surface modulates cellular responses in terms of adhesion, proliferation, inflammatory reactions and survival.

Due to its surface properties an artificial implant usually induces the production of a fibrous tissue covering its surface after implantation. This fibrous layer reduces the tissueimplant contact, which may result in loosening of the implant or stimulation of further inflammatory processes. There are several strategies to enhance the ingrowth of non-biological materials by addressing non-specific cell-surface interactions. Alterations of the physical surface properties like hydrophobicity or surface topography are possibilities. For example, by creating nano-sized features on titanium-based surfaces the expression of bone sialoprotein (BSP) and osteopontin was stimulated by osteogenic cells (2). The enhancement of osteoblast adhesion and function on different nanostructured metal and ceramic materials was demonstrated in further studies $(3,4)$.

A more biomimetic strategy is to control adhesion, proliferation and differentiation of osteogenic cells by biochemical modification of the implant surface. While coating with hydroxyapatite is often used in combination with an alteration of surface roughness to improve osteointegration of implants (5), this approach does not minimize non-specific interactions e.g. interactions with plasma fibronectin that do not necessarily contribute to an optimal bone ingrowth. They may instead lead to negative effects like foreign body reaction and fibrotic processes $(5,6)$. Specific tuning of the material surface can be used to minimize these non-specific effects and may rely on the molecular mechanisms of cell adhesion mainly mediated by integrins which provide selective interactions with the proteins of the extracellular matrix (7). A number of integrin subtypes recognize the tripeptide sequence Arg-Gly-Asp 
(RGD) as their ligand, but show preferential interactions depending on the amino acids flanking the RGD motif as well as on the overall conformation of the peptide (8-11). Therefore, linking of adhesion peptides to biomaterials is a widely used approach to improve biocompatibility, biological activity and the interaction with cells (12-14).

We have previously described a system for surface coating by preparing ultra thin films $(30 \pm 5 \mathrm{~nm})$ of reactive star shaped NCO-sP(EO-stat-PO) macromers with $80 \%$ EO content and isocyanate functional groups at the distal ends (15). These films exhibit minimal interactions with immobilized proteins and allow the reversible folding of the immobilized proteins $(16,17)$. Unmodified NCO-sP(EO-stat-PO) coatings prevent non-specific protein binding and the adhesion of cells under standard cell culture conditions over at least 4 weeks. This feature also reduces bacterial adhesion and creates additional antimicrobial properties (18). Because of the unique switch from the chemically reactive isocyanate groups to an inert polymer layer, during the NCO-sP(EO-stat-PO) layer preparation, an easy integration of cell-binding supporting RGD peptides was demonstrated (19).

For specific functionalization of implant surfaces the preservation of sequence- and conformation-dependent biochemical properties of the peptides is necessary. Therefore, the primary aim of this work was to investigate if the microenvironment of the NCO-sP(EO-stat-PO) layer influences the functional properties of the covalently immobilized adhesion peptides. Secondly, we wanted to establish a substrate-specific experimental approach for testing different adhesion peptides without interference from non-specific protein binding, which occurs in most of the experimental setups used so far. To address these aims we compared linear RGD (gRGDsc) peptides with cyclic RGD (RGDfK) peptides that were integrated in an NCO-sP(EO-stat-PO)-coated surface, with respect to cell adhesion, proliferation and differentiation of bone marrow-derived multipotent mesenchymal stromal cells (MSC) and SaOS-2 cells.

\section{Materials and methods}

Preparation of $N C O$-sP(EO-stat-PO)-coated surfaces. A six-arm star-shaped polymer with a molecular weight of $12,000 \mathrm{~g} / \mathrm{mol}$ was used in this study. The preparation of the star polymers has been described elsewhere (17). Glass substrates $(\mathrm{d}=18 \mathrm{~mm})$ were coated as described earlier (19).

To obtain films that are modified with RGD peptides, the respective amount of linear (gRGDsc, $2 \mathrm{mg} / \mathrm{ml}$ THF) or cyclic RGD (RGDfK, $1 \mathrm{mg} / \mathrm{ml}$ THF) was dissolved in $9 \mathrm{ml}$ of deionised water. This solution was mixed with the star polymer solution in $1 \mathrm{ml}$ THF. The film formation was then done as described (19). The linear gRGDsc peptide was provided by Harm-Anton Klok (EPFL Lausanne) and the cyclic RGDfK peptide was prepared as previously described (20).

Cell culture. MSC were harvested from human bone marrow derived from routine surgical procedures with informed consent from six patients (age 19-30 years) in accordance with the terms of the ethics committee of the University of Ulm.

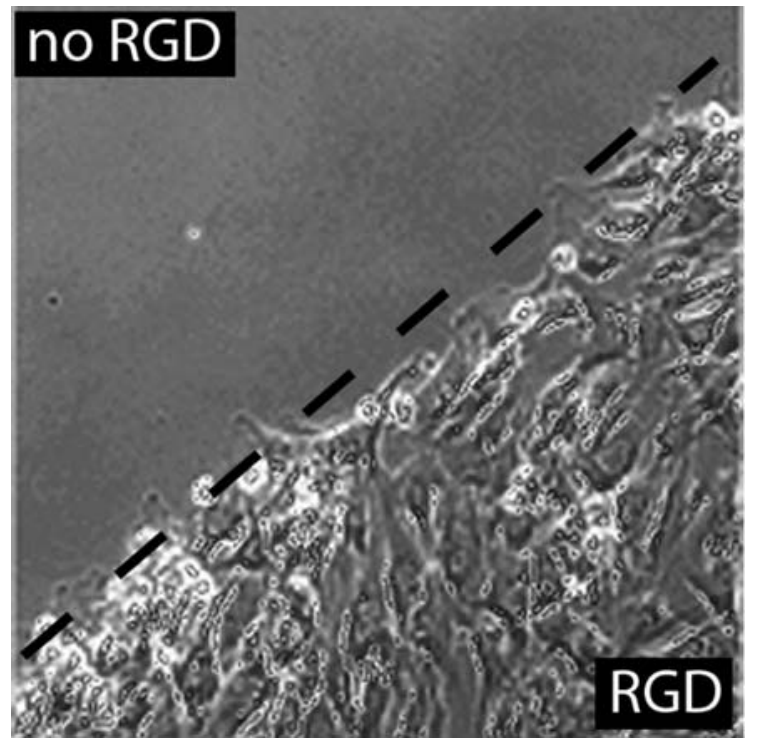

Figure 1. Cultivation of MSC on NCO-sP(EO-stat-PO)-RGD coated surfaces. After covering of the glass pane with NCO-sP(EO-stat-PO), half of the same substrate was dip-coated with NCO-sP(EO-stat-PO) containing cyclic RGD. A sharp borderline of attached cells to the non-adhesive part was detectable. The border is marked with a dotted line only on the picture and was not visible on the substrate (microscopic view, magnification x100).

Isolation and cultivation were done as described earlier (21). The preparation is specific for adherent mesenchymal progenitor cells and maintains the progenitor phenotype. After isolation, the cells were cultured in a basal medium (BM) consisting of DMEM with $10 \%$ FBS, $1 \%$ glutamine and $1 \%$ penicillin/streptomycin (Biochrom-Seromed, Berlin, Germany) at $37^{\circ} \mathrm{C}, 5 \% \mathrm{CO}_{2}$ in $95 \%$ humidity. In order to differentiate the MSC into osteoblasts, the basal medium was supplemented with $10^{-7} \mathrm{M}$ dexamethasone, $50 \mu \mathrm{g} / \mathrm{ml}$ ascorbic acid and $2.16 \mu \mathrm{g} / \mathrm{ml}$ ß-glycerophosphate (Sigma, Germany) for 14 days (differentiation medium, DM). The medium was changed twice a week. The osteogenic differentiation was assessed by RT-PCR of osteoblast-specific genes.

The osteoblastic cells were cultivated under the same conditions in DMEM with $10 \%$ FBS, $1 \%$ penicillin/streptomycin and ascorbic acid $50 \mathrm{mg} / \mathrm{l}$.

RT-PCR analysis. The phenotypic status of the MSC, the differentiated progenitor cells, and osteoblasts was affirmed by RT-PCR analysis of the following osteoblastic markers: alkaline phosphatase (AP), osteocalcin (OC), BSP, RUNX2 (RX2) and osteonectin (ON; SPARC). Collagen type I $\alpha 1$ (COL1A1), and the housekeeping gene glyceraldehyde-3phosphate dehydrogenase (GAPDH) were used to verify the PCR results as internal standards. Therefore, total RNA was isolated from $10^{5}$ cells with the RNeasy ${ }^{\circledR}$ system and reverse transcription was done with Omniscript ${ }^{\mathrm{TM}}$ RT kit (Qiagen, Hilden, Germany) following the manufacturer's instructions. PCR-primers for GAPDH, COL1A1, AP, OC, RX2, and ON were described elsewhere (21). Amplicon size was set to 180-250 bp for GAPDH, COL1A1, AP, OC, RX2, and to $600 \mathrm{bp}$ for ON and BSP. Temperature optimum was set to $60^{\circ} \mathrm{C}$. In all cases, the best primer pair was used. PCR reactions were performed with a Robocycler ${ }^{\circledR}$ (Stratagene, Amsterdam, 

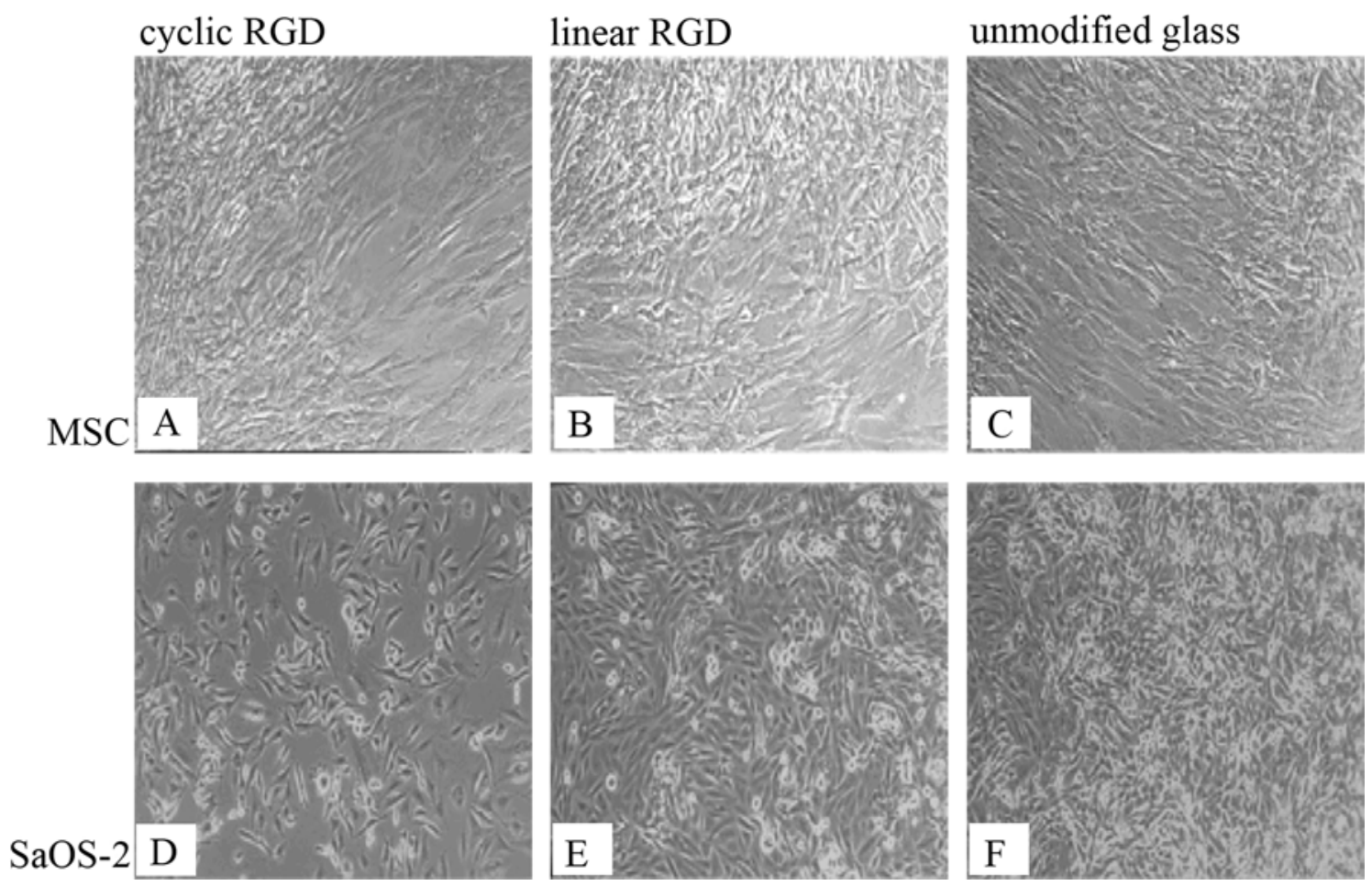

Figure 2. Cell attachment on different NCO-sP(EO-stat-PO)-RGD coatings and unmodified glass panes. No significant differences between linear and cyclic RGD coatings could be detected concerning cell attachment of MSC and SaOS-2 cells (microscopic view, magnification x100).

The Netherlands) using the HotStarTaq ${ }^{\mathrm{TM}}$ Master Mix kit (Qiagen). PCR was performed under linear conditions using the cycle profile: initial incubation for $15 \mathrm{~min}$ at $95^{\circ} \mathrm{C}$, followed by 30 cycles of annealing for $45 \mathrm{sec}$ at $60^{\circ} \mathrm{C}$, extension for $45 \mathrm{sec}$ at $72^{\circ} \mathrm{C}$ and denaturation for $60 \mathrm{sec}$ at $94^{\circ} \mathrm{C}$ and termination for $15 \mathrm{~min}$ at $72^{\circ} \mathrm{C}$.

PCR products were separated on a $1.5 \%$ agarose gel and stained with ethidium bromide, visualized and a digital image obtained with the Gel Doc XR system (Bio-Rad, München, Germany).

Detection of cell adhesion. Cell attachment and adhesion on modified glass substrates was controlled by light microscopy using a Zeiss 'Axioskop mot plus' (Zeiss, Oberkochen, Germany) microscope with a Zeiss AxioCam MRc and the Zeiss AxioVision (v. 3.1) software.

Thiazolyl blue tetrazolium bromide (MTT) assay. MTT assays were carried out as follows: cells were trypsinized, counted, and seeded on NCO-sP(EO-stat-PO)-coated plates placed in 6-well plates. After 1 or 2 weeks, $3 \mathrm{ml}$ of the culture medium were supplemented 1:40 with MTT solution $(5 \mathrm{mg} / \mathrm{ml})$ and the plates were returned to the $37^{\circ} \mathrm{C}$ incubator for $2 \mathrm{~h}$. Afterward, the supernatant was aspirated from each well, and replaced by $1 \mathrm{ml} 0.04 \mathrm{M} \mathrm{HCl} /$ isopropanol. The plates were then incubated for $5 \mathrm{~min}$ at $37^{\circ} \mathrm{C}$. Subsequently, the optical density of $200 \mu 1$ samples was measured at $590 \mathrm{~nm}$ using an ELISA reader (Dynatech Laboratories, USA).

\section{Results}

We were able to show in our present study that an NCO$\mathrm{sP}(\mathrm{EO}-$ stat-PO)-coated surface functionalized with linear or cyclic RGD peptides, preserving the different biochemical and biological properties of the bound peptides. Additionally, a specific, cell type-dependent, influence of the two tested peptides was shown.

The osteogenic differentiated MSC (dOB) and the SaOS-2 cells showed different responses in terms of cell adhesion, proliferation, and osteogenic differentiation on NCO-sP(EOstat-PO)-RGD-coated surfaces.

Cell adhesion on linear and cyclic RGD peptide surfaces. MSC and SaOS-2 were seeded on NCO-sP(EO-stat-PO)coated glass plates that were functionalized with different RGD peptides (Fig. 1). As shown the cells could not cover areas without RGD supplementation. Cell adhesion was detectable only in the presence of linear- or cyclic-RGDs. The principal cell adhesion properties of the cyclic RGDfK have been previously described $(22,23)$. In the present study, cell adhesion on linear and cyclic RGD-modified surfaces showed no obvious differences (Fig. 2). Both MSC and $\mathrm{SaOS}-2$ cells could adhere on the functionalized NCO-sP(EOstat-PO) layers. Additionally, after $24 \mathrm{~h}$ of cultivation no differences concerning the cell shape, number of attached cells and percent coverage of the substrates were detectable on unmodified glass and NCO-sP(EO-stat-PO)-RGD surfaces. Obviously, the integrated amount of RGD peptides could substitute the non-specific adhesive protein binding on the unmodified glass surfaces.

The time span between cell seeding and cell attachment, however, was faster on the NCO-sP(EO-stat-PO)-RGD surfaces compared to glass surfaces without modification. During the 28 days of cultivation, MSC, dOB and SaOS-2 proliferated and covered the complete area of the substrates (Fig. 3). Cell morphology did not change during cultivation. 

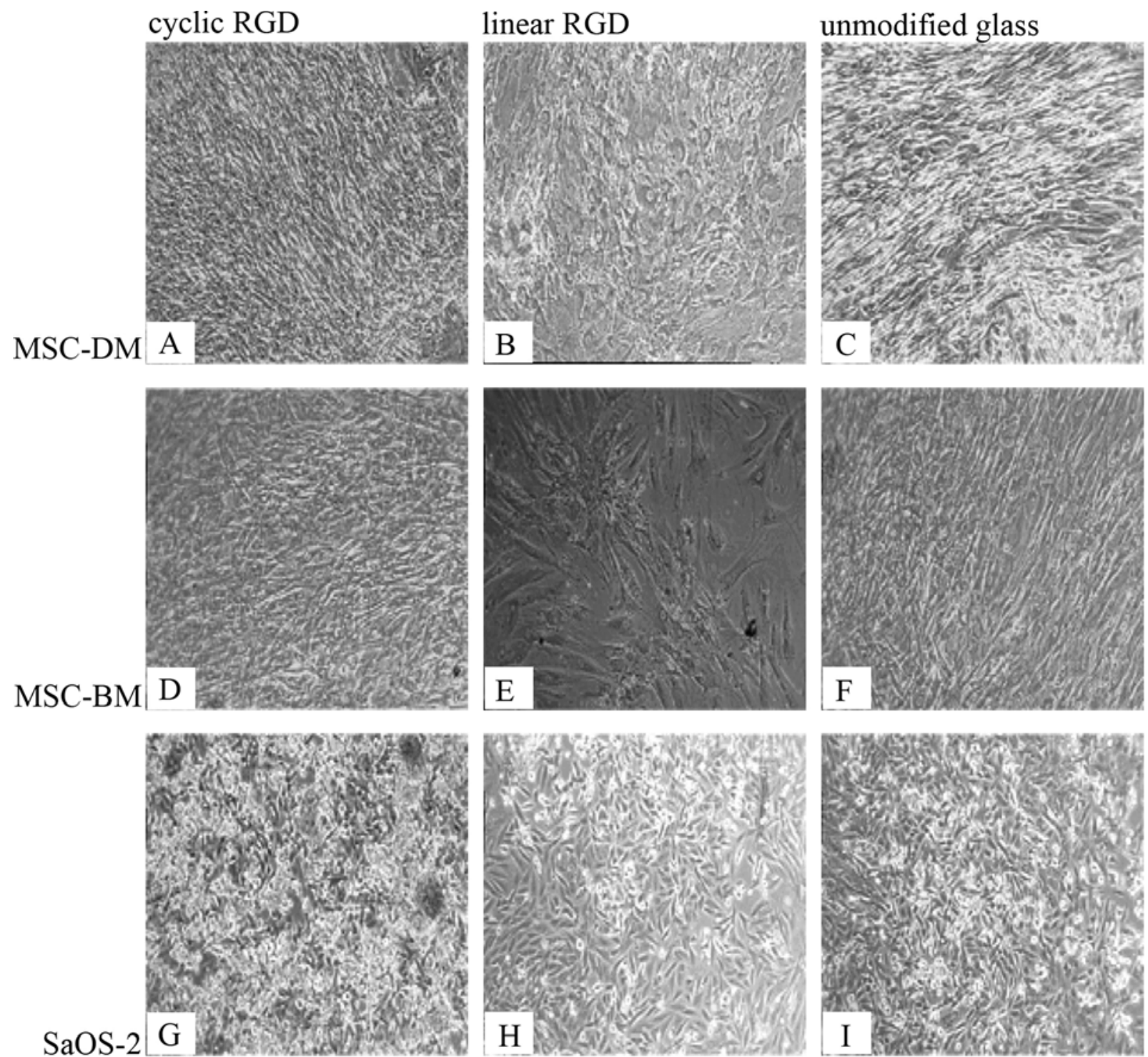

Figure 3. Differentiation and growth of MSC during 28 days of cultivation. DOB (differentiated osteoblasts) MSC (without differentiation conditions). (A) MSC with DM (differentiation medium) on cyclic-RGD-substrate [1 $\mathrm{mg}$ cyclic-RGD/ml NCO-sP(EO-stat-PO) = cyclic RGD]. (B) MSC with DM on linearRGD-substrate [2 mg linear-RGD/ml NCO-sP(EO-stat-PO) = linear RGD]. (C) MSC with DM on glass without coating. (D) MSC with BM (basal medium) on cyclic RGD. (E) MSC with BM on linear RGD. (F) MSC with BM on glass. (G) SaOS-2 with BM on cyclic RGD. (H) SaOS-2 with BM on linear RGD. (I) SaOS-2 on glass.

No signs of enhanced cell death or cellular detachment were detectable.

MTT-test. Cell proliferation testing was done by using the MTT-test comparing the proliferation rate of cells cultivated on commercial cell culture material with cells grown on NCO-sP(EO-stat-PO)-RGD surfaces. The influence of the NCO-sP(EO-stat-PO)-RGD coatings and cultivation conditions is apparent (Fig. 4). On cyclic RGD surfaces MSC proliferated under differentiation conditions (MSC-DM) 4 times faster as MSC-BM and SaOS-2 cells. In contrast, on linear RGD surfaces MSC proliferated 5 times faster under basal conditions (MSC-BM), which was also the case for cultivation on native glass surfaces.

Differentiation of cells on functionalized surfaces. In order to test if the NCO-sP(EO-stat-PO)-coated surfaces, which were

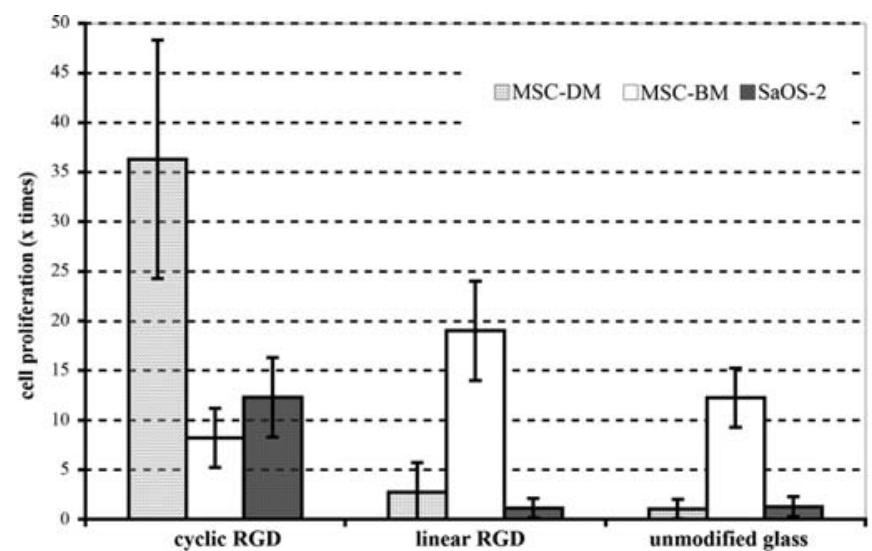

Figure 4. Cell vitality and proliferation on different surface coatings tested by the MTT assay. Cell proliferation was compared to the cell number when seeded $($ mean \pm SD) $(n=3)$. 
$\begin{array}{lllllllllllllllllllllllll}1 & 2 & 3 & 4 & 5 & 6 & 7 & 8 & 1 & 2 & 3 & 4 & 5 & 6 & 7 & 8 & 1 & 2 & 3 & 4 & 5 & 6 & 7 & 8\end{array}$
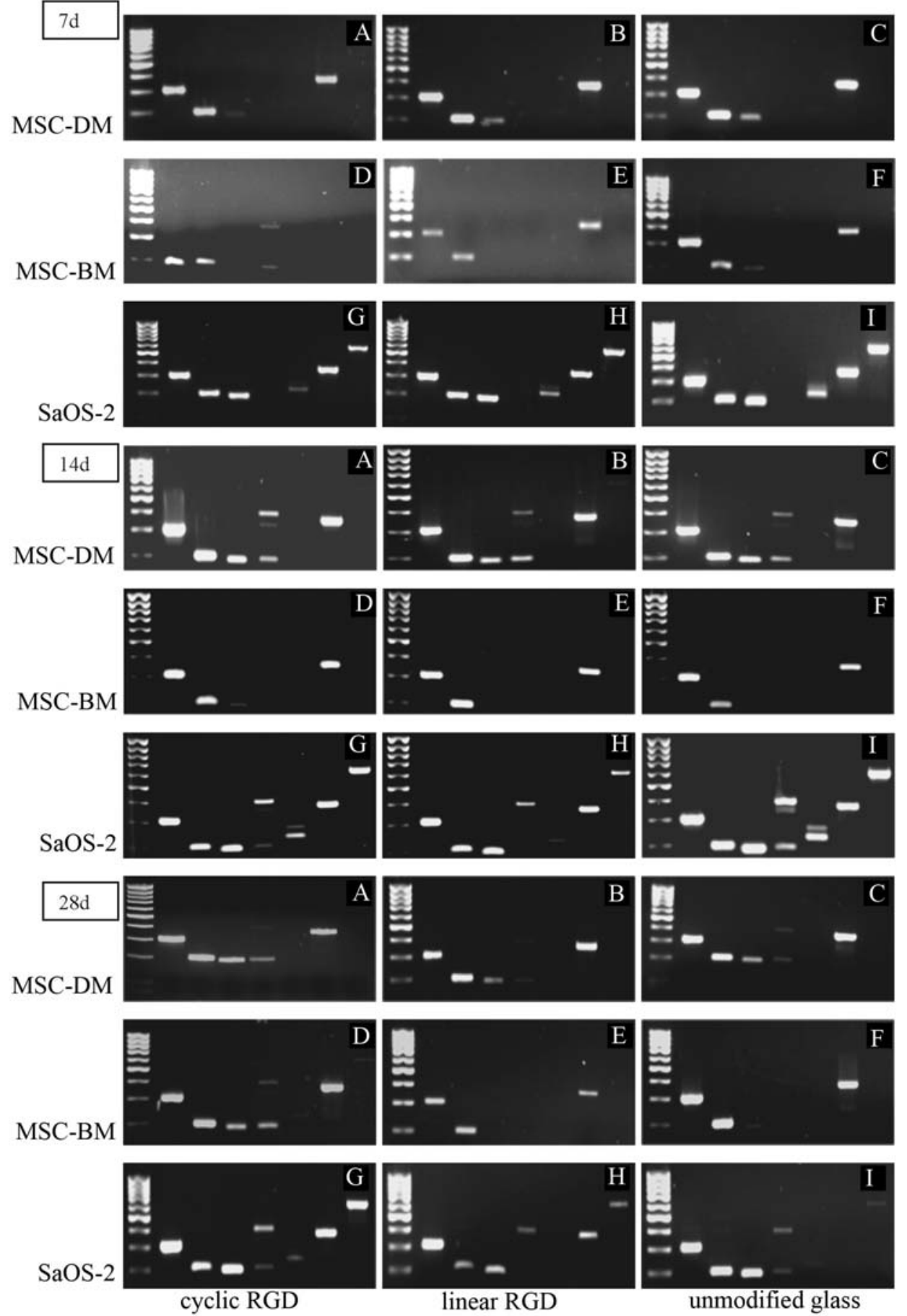

Figure 5. Osteogenic marker gene expression in MSC and SaOS-2. After 7, 14, and 21 days gene expression was assessed by RT-PCR. (A) MSC with differentiation medium (DM) on cyclic-RGD-substrate $[1 \mathrm{mg}$ cyclic-RGD/ml NCO-sP(EO-stat-PO) = cyclic RGD]. (B) MSC with DM on linear-RGDsubstrate $[2 \mathrm{mg}$ linear-RGD/ml NCO-sP(EO-stat-PO) = linear RGD]. (C) MSC with DM on glass surface without NCO-sP(EO-stat-PO)-RGD. (D) MSC with BM (basal medium) on cyclic RGD. (E) MSC with BM on linear RGD. (F) MSC with BM on glass surface. (G) SaOS-2 with BM on cyclic RGD. (H) SaOS-2 with BM on linear RGD. (I) SaOS-2 with BM on glass. Lane 1, 100 bp DNA-ladder; 2, GAPDH; 3, collagen type I $\alpha$ 1; 4, alkaline phosphatase; 5, osteocalcin; 6, RUNX 2; 7, osteonectin; 8, bone sialoprotein.

functionalized with the linear (gRGDsc) or cyclic (RGDfK) peptides influence the osteogenic differentiation potential of MSC, different cultivation conditions were used. After 1, 2, and 3 weeks, the gene expression of osteogenic markers from MSC under basal conditions (MSC-BM), MSC under differentiation conditions (MSC-DM), and SaOS-2 cells under basal conditions was analyzed. In Fig. 5 the gene expression of osteogenic marker genes in cells cultivated on NCO-sP(EOstat-PO)-coated surfaces with linear or cyclic RGD peptides and on unmodified glass as a control, is shown. We observed that under basal conditions and after 28 days on cyclic RGDcoated surfaces MSC express the osteogenic markers AP and OC, while they did not express these markers on surfaces functionalized with linear RGD or cultivated on glass surfaces. 
Nevertheless, under differentiation conditions osteogenic marker gene expression was noted in MSC on all tested surfaces. The expression of osteoblastic markers in SaOS-2 cells was preserved on surfaces coated with linear or cyclic RGD.

\section{Discussion}

Our studies with MSC, MSC differentiated into osteoblasts and SaOS-2 cells, showed that cell adhesion, spreading, and cell vitality on linear gRGDsc and cyclic RGDfK modified $\mathrm{NCO}-\mathrm{sP}(\mathrm{EO}-$ stat-PO) surfaces were significantly modulated by the presented peptides. The results confirmed that an effective covalent surface modification was achieved and we demonstrated that the coating conserves the biochemical properties of these different RGD peptides. The production process did not impair cell adhesion nor inhibit osteogenic differentiation. Using established cell culture protocols MSC could be differentiated into osteoblast-like cells in contact with the RGD-functionalized substrates. Negative effects from the NCO-sP(EO-stat-PO) coating on cell adhesion and differentiation were not observed.

Within the first hour after cell seeding the majority of the cells attached to both RGD peptides correlated with a strong cell spreading, while the cell attachment on unmodified glass surfaces was slower and corresponded to a round cell shape. This probably reflects the different amount of presented binding sites. In order to attach to biomaterial surfaces a cell has to flatten and spread its plasma membrane over the substratum, which is indicative of a high number of binding sites on the material surface $(24,25)$. Coating with NCOsP(EO-stat-PO)-RGD reduced the time for cell adhesion, because of the immediate availability of sufficient cell binding motifs described by several studies $(26,27)$. This is important, because NCO-sP(EO-stat-PO) without functionalization, inhibits cell adhesion by preventing any non-specific protein binding to coated surfaces. Therefore, the addition of cell binding molecules is required in order to use such coatings for biomaterials that have to be integrated into tissues (16). The unmodified glass surfaces first have to be passively coated by a sufficient amount of adhesive proteins, derived from the supplemented serum (e.g. fibronectin) or secreted by the cells themselves, to allow cell adhesion. This may be the major reason for the longer time period necessary to attach and spread on the uncoated glass surface.

The linear and cyclic RGD peptide integration differentially modified the proliferation rate of the different cell types as shown by the MTT assay. While it has been previously reported, that both the osteogenic differentiation of MSC $(28,29)$ and the interaction with different biomaterials affect cell proliferation (30), it has not been described that the physio-chemical properties of linear and cyclic RGD peptides also influence the proliferation rate of human MSC depending on the presence or absence of conditions inducing osteogenic differentiation. This may partly be explained by the fact that the cyclic RGD pentapeptides have higher binding affinity and selectivity for $\alpha \mathrm{v} \beta 3, \alpha \mathrm{v} \beta 5$ and to a much lesser extent for $\alpha I I b \beta 3$ integrin receptors (31-33). Human MSC express $\alpha \mathrm{v} ß 3$ and $\alpha \mathrm{v} \beta 5$ integrin receptors (34) and these receptors have been implicated in the functional regulation of osteogenic cells (35).
In the present study, the NCO-sP(EO-stat-PO) surface functionalized with linear RGD peptides did not induce or inhibit osteogenic differentiation of MSC and maintained the differentiation status of SaOS-2 cells. This is in accordance with previously published results (19). The cyclic-RGDfunctionalized surfaces supported osteogenic differentiation of human MSC even under basal conditions. This may at least partly be explained by the more rigid binding through $\alpha \mathrm{v} ß 3$ and $\alpha \mathrm{v} ß 5$ integrin receptors since associated effects on the cytoskeleton might contribute to the fate determination (36). Our results are in agreement with a recent study on the osteogenic differentiation of MSC in a 3-dimensional alginate matrix in which cyclic and linear RGD peptides were incorporated (37). In contrast, Yang et al reported, that incorporation of linear RGD adhesive peptides in a polyethylene glycol diacrylate hydrogel stimulates osteogenesis of bone marrow stromal cells (38). Possibly, the 3-dimensional cultivation conditions used by Yang et al contributed to this opposite result since we used a 2-dimensional system. The interaction of adhesive ligands and their spatial presentation clearly deserves further investigation.

Taken together, our studies on cell adhesion and cell shape showed that cell adhesion on linear or cyclic RGDfunctionalized NCO-sP(EO-stat-PO) coatings is mediated by specific interactions between the RGD peptides, which were covalently linked to the polymer and the corresponding integrin receptors on the cell surface. The NCO-sP(EO-statPO) coating did not affect the biological and chemical properties of the integrated linear or cyclic RGD peptides. This is supported by the higher MSC proliferation under differentiating conditions and the induction of osteogenic differentiation under basal conditions on cyclic RGD modified coatings. Therefore, ultrathin coatings with the NCO-sP(EOstat-PO) system and specific functionalization with adhesive peptides represent a promising strategy to improve cellbiomaterial interactions at the bio-interface (39). The NCO$\mathrm{sP}(\mathrm{EO}$-stat-PO) system proved to be a suitable tool to investigate the effect of integrated adhesion peptides with minimization of the non-specific interactions with secondary adsorbed proteins on surfaces.

\section{Acknowledgements}

The authors thank Harm-Anton Klok (EPFL Lausanne) for donation of the linear gRGDsc peptide sequence. The VWfoundation and the DFG (TR-SFB 37, Project B1) are gratefully acknowledged for funding.

\section{References}

1. Salata O: Applications of nanoparticles in biology and medicine. J Nanobiotechnology 2: 3, 2004.

2. Gutwein LG and Webster TJ: Increased viable osteoblast density in the presence of nanophase compared to conventional alumina and titania particles. Biomaterials 25: 4175-4183, 2004.

3. Sjöström T, Dalby MJ, Hart A, Tare R, Oreffo ROC and Su B: Fabrication of pillar-like titania nanostructures on titanium and their interactions with human skeletal stem cells. Acta Biomater 5: 1433-1441, 2009.

4. Sato M, Aslani A, Sambito MA, Kalkhoran NM, Slamovich EB and Webster TJ: Nanocrystalline hydroxyapatite/titania coatings on titanium improves osteoblast adhesion. J Biomed Mater Res A 84: $265-272,2008$ 
5. Ramires PA, Romito A, Cosentino F and Milella E: The influence of titania/hydroxyapatite composite coatings on in vitro osteoblasts behaviour. Biomaterials 22: 1467-1474, 2001

6. Keselowsky BG, Bridges AW, Burns KL, et al: Role of plasma fibronectin in the foreign body response to biomaterials. Biomaterials 28: 3626-3631, 2007.

7. Hynes RO: Integrins: bidirectional, allosteric signaling machines. Cell 110: 673-687, 2002.

8. Villard V, Kalyuzhniy O, Riccio O, et al: Synthetic RGDcontaining alpha-helical coiled coil peptides promote integrindependent cell adhesion. J Pept Sci 12: 206-212, 2006.

9. Ochsenhirt SE, Kokkoli E, McCarthy JB and Tirrell M: Effect of RGD secondary structure and the synergy site PHSRN on cell adhesion, spreading and specific integrin engagement. Biomaterials 27: 3863-3874, 2006.

10. Garcia AJ, Schwarzbauer JE and Boettiger D: Distinct activation states of alpha5beta1 integrin show differential binding to RGD and synergy domains of fibronectin. Biochemistry 41: 9063-9069, 2002.

11. Petrie TA, Capadona JR, Reyes CD and Garcia AJ: Integrin specificity and enhanced cellular activities associated with surfaces presenting a recombinant fibronectin fragment compared to RGD supports. Biomaterials 27: 5459-5470, 2006.

12. Heckmann L, Fiedler J, Mattes T and Brenner RE: Mesenchymal progenitor cells communicate via alpha and beta integrins with a three-dimensional collagen type I matrix. Cells Tissues Organs 182: 143-154, 2006.

13. Keselowsky BG, Collard DM and Garcia AJ: Integrin binding specificity regulates biomaterial surface chemistry effects on cell differentiation. Proc Natl Acad Sci USA 102: 5953-5957, 2005.

14. Hubbell JA: Tissue and cell engineering. Curr Opin Biotechnol 14: 517-519, 2003

15. Groll J, Haubensak W, Ameringer T and Moeller M: Ultrathin coatings from isocyanate terminated star PEG prepolymers: patterning of proteins on the layers. Langmuir 21: 3076-3083, 2005.

16. Amirgoulova EV, Groll J, Heyes CD, et al: Biofunctionalized polymer surfaces exhibiting minimal interaction towards immobilized proteins. Chemphyschem 5: 552-555, 2004.

17. Groll J, Amirgoulova EV, Ameringer T, et al: Biofunctionalized, ultrathin coatings of cross-linked star-shaped poly(ethylene oxide) allow reversible folding of immobilized proteins. J Am Chem Soc 126: 4234-4239, 2004

18. Groll J, Fiedler J, Bruellhoff K, Moeller M and Brenner RE: Novel surface coatings modulating eukaryotic cell adhesion and preventing implant infection. Int J Artif Organs 32: 655-662, 2009.

19. Groll J, Fiedler J, Engelhard E, et al: A novel star PEG-derived surface coating for specific cell adhesion. J Biomed Mater Res A 74: 607-617, 2005.

20. Kantlehner M, Schaffner P, Finsinger D, et al: Surface coating with cyclic RGD peptides stimulates osteoblast adhesion and proliferation as well as bone formation. Chembiochem 1: 107-114, 2000 .

21. Fiedler J, Roderer G, Gunther KP and Brenner RE: BMP-2, BMP-4, and PDGF-bb stimulate chemotactic migration of primary human mesenchymal progenitor cells. J Cell Biochem 87: 305-312, 2002

22. Auernheimer J, Zukowski D, Dahmen C, et al: Titanium implant materials with improved biocompatibility through coating with phosphonate-anchored cyclic RGD peptides. Chembiochem 6: 2034-2040, 2005.

23. Lieb E, Hacker M, Tessmar J, et al: Mediating specific cell adhesion to low-adhesive diblock copolymers by instant modification with cyclic RGD peptides. Biomaterials 26: 2333-2341, 2005.
24. Allen LT, Tosetto M, Miller IS, et al: Surface-induced changes in protein adsorption and implications for cellular phenotypic responses to surface interaction. Biomaterials 27: 3096-3108, 2006.

25. Owen GR, Meredith DO, ap Gwynn I and Richards RG: Focal adhesion quantification - a new assay of material biocompatibility? Review. Eur Cell Mater 9: 85-96, 2005.

26. Hersel U, Dahmen C and Kessler H: RGD modified polymers: biomaterials for stimulated cell adhesion and beyond. Biomaterials 24: 4385-4415, 2003.

27. Koblinski JE, Wu M, Demeler B, Jacob K and Kleinman HK: Matrix cell adhesion activation by non-adhesion proteins. J Cell Sci 118: 2965-2974, 2005

28. Mata A, Boehm C, Fleischman AJ, Muschler G and Roy S: Growth of connective tissue progenitor cells on microtextured polydimethylsiloxane surfaces. J Biomed Mater Res 62: 499-506, 2002.

29. Muschler GF, Midura RJ and Nakamoto C: Practical modeling concepts for connective tissue stem cell and progenitor compartment kinetics. J Biomed Biotechnol 2003 (3): 170-193, 2003.

30. Gravel M, Gross T, Vago R and Tabrizian M: Responses of mesenchymal stem cell to chitosan-coralline composites microstructured using coralline as gas forming agent. Biomaterials 27: 1899-1906, 2006

31. Pfaff M, Tangemann K, Muller B, et al: Selective recognition of cyclic RGD peptides of NMR defined conformation by alpha IIb beta 3 , alpha $\mathrm{V}$ beta 3 , and alpha 5 beta 1 integrins. J Biol Chem 269: 20233-20238, 1994.

32. Haubner R, Gratias R, Diefenbach B, Goodman SL, Jonczyk A and Kessler H: Structural and functional aspects of RGDcontaining cyclic pentapeptides as highly potent and selective integrin alphaVbeta3 antagonists. J Am Chem Soc 118: 7461-7472, 1996.

33. Aumailley M, Gurrath M, Müller G, Calvete J, Timpl R and Kessler H: Arg-Gly-Asp constrained within cyclic pentapeptides. Strong and selective inhibitors of cell adhesion to vitronectin and laminin fragment P1. FEBS Lett 291: 50-54, 1991.

34. Docheva D, Popov C, Mutschler W and Schieker M: Human mesenchymal stem cells in contact with their environment: surface characteristics and the integrin system. J Cell Mol Med 11: 21-38, 2007.

35. Schaffner P and Dard MM: Structure and function of RGD peptides involved in bone biology. Cell Mol Life Sci 60: 119-132, 2003.

36. Treiser MD, Yang EH, Gordonov S, et al: Cytoskeleton-based forecasting of stem cell lineage fates. Proc Natl Acad Sci USA 107: 610-615, 2010.

37. Hsiong SX, Boontheekul T, Huebsch N and Mooney DJ: Cyclic arginine-glycine-aspartate peptides enhance three-dimensional stem cell osteogenic differentiation. Tissue Eng Part A 15: 263-272, 2009.

38. Yang F, Williams CG, Wang DA, Lee H, Manson PN and Elisseeff J: The effect of incorporating RGD adhesive peptide in polyethylene glycol diacrylate hydrogel on osteogenesis of bone marrow stromal cells. Biomaterials 26: 5991-5998, 2005.

39. Magdolen U, Auernheimer J, Dahmen C, et al: Growth promoting in vitro effect of synthetic cyclic RGD-peptides on human osteoblast-like cells attached to cancellous bone. Int J Mol Med 17: 1017-1021, 2006. 